\title{
A Tuning Procedure for ARX-based MPC
}

\section{Olesen, Daniel; Huusom, Jakob Kjøbsted; Jørgensen, John Bagterp}

Published in:

2013 IEEE Multi-conference on Systems and Control

Link to article, DOI:

10.1109/CACSD.2013.6663481

Publication date:

2013

Document Version

Publisher's PDF, also known as Version of record

Link back to DTU Orbit

Citation (APA):

Olesen, D., Huusom, J. K., \& Jørgensen, J. B. (2013). A Tuning Procedure for ARX-based MPC. In 2013 IEEE Multi-conference on Systems and Control (pp. 188-193). IEEE. https://doi.org/10.1109/CACSD.2013.6663481

\section{General rights}

Copyright and moral rights for the publications made accessible in the public portal are retained by the authors and/or other copyright owners and it is a condition of accessing publications that users recognise and abide by the legal requirements associated with these rights.

- Users may download and print one copy of any publication from the public portal for the purpose of private study or research.

- You may not further distribute the material or use it for any profit-making activity or commercial gain

- You may freely distribute the URL identifying the publication in the public portal

If you believe that this document breaches copyright please contact us providing details, and we will remove access to the work immediately and investigate your claim 


\title{
A Tuning Procedure for ARX-based MPC
}

\author{
Daniel Haugaard Olesen, Jakob Kjøbsted Huusom, John Bagterp Jørgensen
}

\begin{abstract}
We present an optimization based tuning procedure with certain robustness properties for an offset free Model Predictive Controller (MPC). The MPC is designed for univariate processes that can be represented by an ARX model. The advantage of ARX model representations is that standard system identification techniques using convex optimization can be used for identification of such models from input-output data. The stochastic model of the ARX model identified from input-output data is modified with an ARMA model designed as part of the MPC-design procedure to ensure offset-free control. The ARMAX model description resulting from the extension can be realized as a state space model in innovation form. The MPC is designed and implemented based on this state space model in innovation form. Expressions for the closed-loop dynamics of the unconstrained system is used to derive the sensitivity function of this system. The closed-loop expressions are also used to numerically evaluate absolute integral performance measures. Due to the closed-loop expressions, these evaluations can be done relative quickly. Consequently, the tuning may be performed by numerical minimization of the integrated absolute error subject to the a constraint on the maximum of the sensitivity function. The latter constraint provides a robustness measure that is essential for the procedure.
\end{abstract}

\section{INTRODUCTION}

Model Predictive Control (MPC) has evolved to become an industrial standard in advanced process control [1]. MPC is a control methodology that uses a model of the system to predict the process output over some future horizon. An ARX model representation of the plant may be obtained from input-output data using convex optimization methods. To ensure offset free control, integrators has to be introduced to the plant model in case of persistent unmeasured disturbances and/or plant model mismatch. In such cases, the observer that guarantees offset free control introduces a plant model mismatch. This plant model mismatch complicates the tuning of the controller [2]-[5].

Despite the growing popularity of MPC, a systematic tuning practice has not evolved, and only few guidelines exist. The topic has not been short of research, as there are numerous academic publications on the subject. A comprehensive review of proposed tuning methods is presented in [6] and loop transfer recovery procedures have also been investigated [7], [8]. Our study relies on a closed loop description of the controller and the process model to assess the performance of an MPC with a given tuning. It has previously been proposed to use a closed loop description for synthesis of a MPC by application of robust design

D.H. Olesen and J.B. Jørgensen are with DTU Compute, Technical University of Denmark, DK-2800 Kgs Lyngby, Denmark. Corresponding author: J.B. Jørgensen, jbjo@dtu . dk

J.K. Huusom is with DTU Chemical Engineering, Technical University of Denmark, DK-2800 Kgs Lyngby, Denmark techniques [9]. In this paper, we state the tuning problem as an inequality constrained optimization problem. We have defined a deterministic tuning objective related to the integrated absolute error and use a bound on the maximum sensitivity to ensure robustness. The objective is defined for a set point change where a gain mismatch is introduced between the observer and our design model. The mismatch is used to ensure that offset free control is obtained.

The paper is organized as follows. Section II describes an ARX-based MPC for univariate processes and develops a state-space model for the controller. Section III derives a state-space model for the closed-loop system and uses this state space model for covariance computation, sensitivity function computation and for computation of integrated absolute error measures. These measures and the sensitivity function are used to formulate an optimization problem for selecting the tuning parameters of the MPC. In Section IV the proposed tuning method is demonstrated for three first order processes with different time delays. Conclusions are presented in Section V.

\section{ARX-BASED MPC FOR SISO SYSTEMS}

In this section we derive a state space representation for an unconstrained MPC based on an ARX-model modified with a filtered integrated white noise stochastic model. First, we represent the ARX model as a state space model in innovation form. Subsequently, we use this state space model in innovation form to derive the correct control law for the unconstrained MPC. As the control law is linear, the resulting controller may be represented in a state space form.

\section{A. State Space Model in Innovation Form}

The ARX model

$$
A\left(q^{-1}\right) y_{k}=B\left(q^{-1}\right) u_{k}+\varepsilon_{k}
$$

has been used in a number of MPC applications. The advantage of this model parametrization is that the parameters may be identified using standard system identification techniques based on convex optimization. To have offset-free control from the MPC based on this model, the stochastic part of the model is modified to be a filtered white noise process

$$
\varepsilon_{k}=\frac{1-\alpha q^{-1}}{1-q^{-1}} e_{k}
$$

where $e_{k} \sim \mathcal{N}_{i i d}\left(0, R_{e e}\right)$. The coefficient $\alpha \in[0,1]$ is a design parameter of the MPC.

The modified ARX model can be represented as an ARIMAX model as:

$$
A\left(q^{-1}\right) y_{k}=B\left(q^{-1}\right) u_{k}+\frac{1-\alpha q^{-1}}{1-q^{-1}} e_{k}
$$


By rearrangement the ARIMAX model can be represented as an ARMAX model:

$$
\bar{A}\left(q^{-1}\right) y_{k}=\bar{B}\left(q^{-1}\right) u_{k}+\bar{C}\left(q^{-1}\right) e_{k}
$$

with

$$
\begin{aligned}
& \bar{A}\left(q^{-1}\right)=\left(1-q^{-1}\right) A\left(q^{-1}\right) \\
& \bar{B}\left(q^{-1}\right)=\left(1-q^{-1}\right) B\left(q^{-1}\right) \\
& \bar{C}\left(q^{-1}\right)=1-\alpha q^{-1}
\end{aligned}
$$

Denote the coefficients of $\bar{A}\left(q^{-1}\right)$ and $\bar{B}\left(q^{-1}\right)$ as

$$
\begin{aligned}
& \bar{A}\left(q^{-1}\right)=1+\bar{a}_{1} q^{-1}+\bar{a}_{2} q^{-2}+\ldots+\bar{a}_{n} q^{-n} \\
& \bar{B}\left(q^{-1}\right)=\bar{b}_{1} q^{-1}+\bar{b}_{2} q^{-2}+\ldots+\bar{b}_{n} q^{-n}
\end{aligned}
$$

Then the system (1)-(2) may be represented as a state space model in innovation form

$$
\begin{aligned}
x_{k+1} & =\hat{A} x_{k}+\hat{B} u_{k}+\hat{K} e_{k} \\
y_{k} & =\hat{C} x_{k}+e_{k}
\end{aligned}
$$

with the state space matrices $(\hat{A}, \hat{B}, \hat{K}, \hat{C})$ realized in observer canonical form

$$
\begin{aligned}
& \hat{A}=\left[\begin{array}{c|cccc}
-\bar{a}_{1} & 1 & 0 & 0 & 0 \\
-\bar{a}_{2} & 0 & 1 & 0 & 0 \\
\vdots & \vdots & & \ddots & \vdots \\
-\bar{a}_{n-1} & 0 & 0 & \cdots & 1 \\
\hline-\bar{a}_{n} & 0 & 0 & \cdots & 0
\end{array}\right] \hat{B}=\left[\begin{array}{c}
\bar{b}_{1} \\
\bar{b}_{2} \\
\vdots \\
\vdots \\
\bar{b}_{n-1} \\
\bar{b}_{n}
\end{array}\right] \hat{K}=\left[\begin{array}{c}
\alpha-\bar{a}_{1} \\
-\bar{a}_{2} \\
\vdots \\
-\bar{a}_{n-1} \\
-\bar{a}_{n}
\end{array}\right] \\
& \hat{C}=\left[\begin{array}{cccccc}
1 & 0 & 0 & \cdots & 0
\end{array}\right]
\end{aligned}
$$

\section{B. Unconstrained MPC for State-Space Models in Innova- tion Form}

The filtered state estimation and the one-step prediction may for state space models in innovation form (7) be combined to give the following expressions for computation of the innovation, $e_{k}$ [4]:

$$
\begin{aligned}
\hat{x}_{k \mid k-1} & =\hat{A} \hat{x}_{k-1 \mid k-2}+\hat{B} u_{k-1}+\hat{K} e_{k-1} \\
\hat{y}_{k \mid k-1} & =\hat{C} \hat{x}_{k \mid k-1} \\
e_{k} & =y_{k}-\hat{y}_{k \mid k-1}
\end{aligned}
$$

Initially, $\hat{x}_{0 \mid-1}$ is known and the one-step prediction (8a) is not needed. Knowing the innovation, $e_{k}$, the predictions in the state space model in innovation form may be represented as [4]

$$
\begin{aligned}
\hat{x}_{k+1 \mid k} & =\hat{A} \hat{x}_{k \mid k-1}+\hat{B} \hat{u}_{k \mid k}+\hat{K} e_{k} \\
\hat{x}_{k+1+j \mid k} & =\hat{A} \hat{x}_{k+j \mid k}+\hat{B} \hat{u}_{k+j \mid k}, \quad j=1, \ldots, N-1 \\
\hat{y}_{k+j \mid k} & =\hat{C} \hat{x}_{k+j \mid k}, \quad j=1, \ldots, N
\end{aligned}
$$

It is important to notice the term $\hat{K} e_{k}$ in (9a). This term is important for derivation of the correct control law [4]. Let the objective of the MPC be

$$
\phi=\frac{1}{2} \sum_{j=0}^{N-1}\left\|\hat{y}_{k+j+1 \mid k}-r_{k+j+1 \mid k}\right\|_{2}^{2}+\lambda\left\|\Delta \hat{u}_{k+j \mid k}\right\|_{2}^{2}
$$

in which the second term, $\left\|\Delta \hat{u}_{k+j \mid j}\right\|_{2}^{2}$, is a regularization term. We assume the reference parametrization, $\left\{r_{k+j \mid k}\right\}_{j=1}^{N}=\left\{r_{k}, \ldots, r_{k}\right\}$. The tuning parameters in this objective function is the scalar weight, $\lambda$, which penalizes control signal movement.

The unconstrained MPC is represented as the convex quadratic optimization problem

$$
\min _{\left\{\hat{u}_{k+j \mid j}\right\}_{j=0}^{N-1}}\left\{\phi=\phi\left(\left\{\hat{u}_{k+j \mid j}\right\}_{j=0}^{N-1} ; r_{k}, u_{k-1}, \hat{x}_{k \mid k-1}, e_{k}\right):\right.
$$

which has the solution $U_{k}=\left[\hat{u}_{k \mid k}, \ldots, \hat{u}_{k+N-1 \mid k}\right]$ with [4]

$$
u_{k}=\hat{u}_{k \mid k}=L_{x} \hat{x}_{k \mid k-1}+L_{w} e_{k}+L_{u} u_{k-1}+L_{r} r_{k}
$$

The specific expressions for and derivation of $L_{x}, L_{w}, L_{u}$ and $L_{r}$ are given in [4]. It must be emphasized that most available expressions for linear-quadratic controllers misses the term $L_{w} e_{k}$ that arise due to the term $\hat{K} e_{k}$ in (9a).

Define the controller states as $x_{k}^{c}=\left[\hat{x}_{k \mid k-1} ; u_{k-1}\right]$ such that the unconstrained MPC consisting of (8) and (11) may be represented in the state space form

$$
\begin{aligned}
x_{k+1}^{c} & =A_{c} x_{k}^{c}+B_{c y} y_{k}+B_{c r} r_{k} \\
u_{k} & =C_{c} x_{k}^{c}+D_{c y} y_{k}+D_{c r} r_{k}
\end{aligned}
$$

with

$$
\begin{aligned}
A_{c} & =\left[\begin{array}{rr}
(\hat{A}-\hat{K} \hat{C})+\hat{B}\left(L_{x}-L_{w} \hat{C}\right) & \hat{B} L_{u} \\
L_{x}-L_{w} \hat{C} & L_{u}
\end{array}\right] \\
B_{c y} & =\left[\begin{array}{c}
\hat{K}+\hat{B} L_{w} \\
L_{w}
\end{array}\right] \quad B_{c r}=\left[\begin{array}{c}
\hat{B} L_{r} \\
L_{r}
\end{array}\right] \\
C_{c} & =\left[\begin{array}{ll}
L_{x}-L_{w} \hat{C} & L_{u}
\end{array}\right] \\
D_{c y} & =L_{w} \quad D_{c r}=L_{r}
\end{aligned}
$$

In addition to the model (1), this controller representation depends on the tuning parameters $(\alpha, \lambda)$.

\section{Closed-Loop System ANd Measures}

Let the system be a LTI system in the form

$$
\begin{aligned}
x_{k+1} & =A x_{k}+B u_{k}+E d_{k}+G w_{k} \\
z_{k} & =C x_{k} \\
y_{k} & =z_{k}+v_{k}
\end{aligned}
$$

where $x_{k}$ is the states, $u_{k}$ is the manipulated inputs, $d_{k}$ are unknown disturbances, $w_{k} \sim \mathcal{N}_{i i d}\left(0, R_{w w}\right)$ is stochastic process noise, $z_{k}$ is the uncorrupted outputs, $v_{k} \sim \mathcal{N}_{i i d}\left(0, R_{v v}\right)$ is measurement noise, and $y_{k}$ is the measurements, i.e. the outputs, $z_{k}$, corrupted by measurement noise, $v_{k}$. This model $(A, B, E, G, C)$ is not necessarily identical to the model $(\hat{A}, \hat{B}, \hat{C}, \hat{K})$ used by the MPC.

Using the system model (14) and the MPC state space representation (12), the closed-loop system may be represented as

$$
\begin{aligned}
x_{k+1}^{c l} & =A_{c l} x_{k}^{c l}+B_{w c l} w_{k}+B_{v c l} v_{k}+B_{r c l} r_{k}+B_{d c l} d_{k} \\
z_{k} & =C_{c l} x_{k}^{c l} \\
y_{k} & =C_{c l} x_{k}^{c l}+v_{k} \\
u_{k} & =C_{u c l} x_{k}^{c l}+D_{v c l} v_{k}+D_{r c l} r_{k}
\end{aligned}
$$




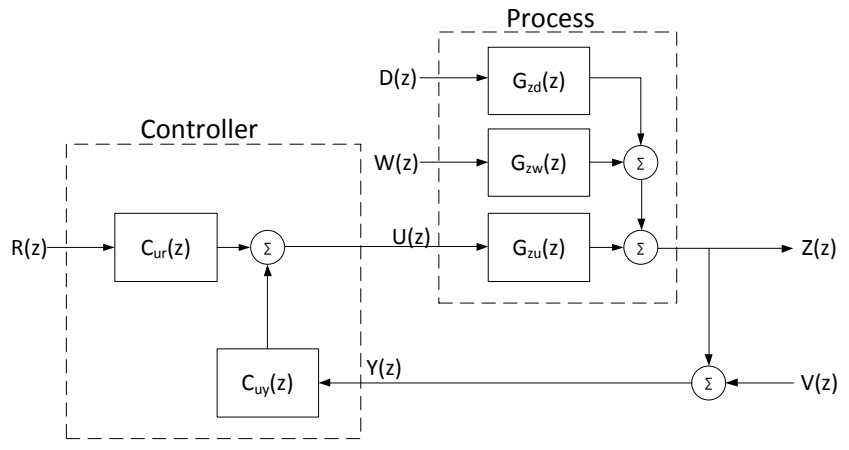

Fig. 1: Closed-loop system. The transfer functions $C_{u r}(z)$ and $C_{u y}(z)$ forms the unconstrained MPC. The controlled outputs of the process is described by $G_{z u}(z), G_{z w}(z)$ and $G_{z d}(z)$.

with $x_{k}^{c l}=\left[x_{k} ; x_{k}^{c}\right]$ and

$$
\begin{aligned}
& A_{c l}=\left[\begin{array}{cc}
A+B D_{c y} C & B C_{c} \\
B_{c y} C & A_{c}
\end{array}\right], B_{w c l}=\left[\begin{array}{c}
G \\
0
\end{array}\right], \\
& B_{v c l}=\left[\begin{array}{c}
B D_{c y} \\
B_{c y}
\end{array}\right], B_{r c l}=\left[\begin{array}{c}
B D_{c r} \\
B_{c r}
\end{array}\right], B_{d c l}=\left[\begin{array}{c}
E \\
0
\end{array}\right], \\
& C_{c l}=\left[\begin{array}{ll}
C & 0
\end{array}\right], C_{u c l}=\left[\begin{array}{ll}
D_{c y} C & C_{c}
\end{array}\right], \\
& D_{v c l}=D_{c y}, D_{r c l}=D_{c r} .
\end{aligned}
$$

This representation depends on the MPC tuning parameters, $(\alpha, \lambda)$, and is used extensively to compute measures for the controller performance. One obvious measure is of course the eigenvalues of the closed-loop system, eig $\left(A_{c l}\right)$. Acceptable tunings must provide stable closed loop systems, i.e. systems in which all the closed-loop eigenvalues of $A_{c l}$ have modulus less than 1.

\section{A. Variance}

The variance of the signals, $\left(z_{k}, y_{k}, u_{k}\right)$, for the closedloop system as response to the exogenous stochastic signals, $w_{k}$ and $v_{k}$, is one measure for the performance of the MPC.

Provided that $A_{c l}$ is stable, the covariance of the states of the closed loop system, $R_{x x}$, may be computed by solution of the discrete Lyapunov equation

$$
R_{x x}=A_{c l} R_{x x} A_{c l}^{T}+B_{w c l} R_{w w} B_{w c l}^{T}+B_{v c l} R_{v v} B_{v c l}^{T}
$$

and the corresponding output variances are

$$
\begin{aligned}
& R_{z z}=C_{c l} R_{x x} C_{c l}^{T} \\
& R_{y y}=C_{c l} R_{x x} C_{c l}^{T}+R_{v v} \\
& R_{u u}=C_{u c l} R_{x x} C_{u c}^{T}+D_{v c l} R_{v v} D_{v c l}^{T}
\end{aligned}
$$

\section{B. Sensitivity}

Fig. 1 illustrates the transfer functions in the closed loop system. The transfer function representation of the process model (14) is

$$
Y(z)=G_{z u}(z) U(z)+G_{z d}(z) D(z)+G_{z w} W(z)+V(z)
$$

and the transfer function of the MPC control law (12) may be represented as

$$
U(z)=C_{u y}(z) Y(z)+C_{u r}(z) R(z)
$$

$G_{z u}(z), G_{z d}(z), G_{z w}(z), C_{u y}(z)$, and $C_{u r}(z)$ may be computed from the associated state-space representations in the standard way. Combining (19) and (20) yields a transfer function for the closed-loop system (15)

$$
Y(z)=S(z) \bar{D}(z)+T(z) R(z)
$$

with $\bar{D}(z)=G_{z d}(z) D(z)+G_{z w}(z) W(z)+V(z)$ and

$$
\begin{aligned}
& S(z)=C_{c l}\left(z I-A_{c l}\right)^{-1} B_{v c l}+1 \\
& T(z)=C_{c l}\left(z I-A_{c l}\right)^{-1} B_{r c l}
\end{aligned}
$$

$S(z)$ is the sensitivity function and $T(z)$ is the complementary sensitivity function. The sensitivity function, $S(z)$, is related to the robustness of the system in relation to modelplant mismatch as well as process and measurement noise [10]. In particular the $\mathcal{H}_{\infty}$ norm of $S(z)$ for a SISO process

$$
M_{S}=\|S(z)\|_{\infty}=\max _{\omega} S\left(e^{j \omega T_{s}}\right)
$$

has been used as a measure of robustness. $T_{s}$ is the sampling time. A common requirement is to select $M_{S} \leq 2$. This corresponds to ensuring a phase margin better than 29 degrees [10].

\section{Integrated Absolute Error}

The integrated absolute error (IAE) is a classical way to measure control systems performance for certain reference and disturbance scenarios of systems without noise $\left(w_{k}=\right.$ 0 and $v_{k}=0$ ). Consider a scenario starting from steady state and specified by $[r(t)]_{t_{0}}^{t_{f}}=\left\{r_{k}\right\}_{k=0}^{n_{f}-1}$ and $[d(t)]_{t_{0}}^{t_{f}}=$ $\left\{d_{k}\right\}_{k=0}^{n_{f}-1}$ with $r(t)=r_{k}$ and $d(t)=d_{k}$ for $t_{k} \leq t<t_{k+1}$ using $t_{k}=t_{0}+k T_{s}$ and $t_{f}=t_{0}+n_{f} T_{s}$. The IAE of this scenario is defined as

$$
\tilde{J}=\int_{t_{0}}^{t_{f}}|y(t)-r(t)| d t
$$

which using Euler integration and $J=\tilde{J} / T_{s}$ may be approximated by

$$
J=\sum_{k=0}^{n_{f}-1}\left|y_{k}-r_{k}\right|
$$

(25) is evaluated by simulation using the deterministic part of (15), the initial steady state, $x_{0}^{c l}=0$, and the specified scenario, $\left\{r_{k}\right\}_{k=0}^{n_{f}-1}$ and $\left\{d_{k}\right\}_{k=0}^{n_{f}-1}$.

In this paper, we assume that $G_{z u}(s)$ are available from the system identification step, while $G_{z w}(s)$ and $G_{z d}(s)$ are not available. Consequently, the scenario simulation can be conducted for reference scenarios only. To have offset free control for the resulting tuning, unknown disturbances are emulated by model-plant mismatch. The model-plant mismatch is conducted using the identified model for the controller design, $\hat{G}_{z u}(s)$, and using a perturbation of this model for the plant. A number of scenarios can be simulated by selecting the plant model from an ensemble of models. 


\section{Tuning}

In the tuning of the MPC, the control and prediction horizon, $N$, is chosen sufficiently large such that the resulting controller for all practical purposes corresponds to an infinity horizon controller, i.e. $u_{k}=\mu_{N}\left(\hat{x}_{k \mid k-1}, e_{k}, u_{k-1}, r_{k}\right) \approx$ $\mu_{\infty}\left(\hat{x}_{k \mid k-1}, e_{k}, u_{k-1}, r_{k}\right)$. The remaining tuning parameters, $(\alpha, \lambda)$, are chosen by solution of the constrained optimization problem

$$
\begin{array}{ll}
\min _{\alpha, \lambda} & J=J(\alpha, \lambda) \\
\text { s.t. } & M_{S}(\alpha, \lambda) \leq M_{S, \max } \\
& 0 \leq \alpha \leq 1 \\
& \lambda_{\min } \leq \lambda \leq \lambda_{\max }
\end{array}
$$

The objective minimizes some measure related to the IAE of the chosen scenarios. The scenarios are created by using the estimated model for the controller design and different model parametrization chosen from an ensemble of models to represent the plant. This model-plant mismatch can be used to represent unknown disturbances. In such scenarios, it is necessary that the controller is equipped with an integrator to provide offset free control. Therefore, instead of evaluating a disturbance scenario, we consider a set-point change scenario where we use a modified gain for the known process model $(A, B, C)$ in $(15)$. We require a modified process model for (15) in order to ensure, an offset free controller is generated from (26). Using the correct model would result in a situation in which the output predictions match the process model and thus eliminating the need for integration, i.e. leading to $\alpha=1$.

It is critical for the usefulness of the resulting tuning that the robustness constraint (26b) is included in the optimization problem. Useless results are obtained if the robustness constraint (26b) is discarded by using a large upper bound. In such cases, the resulting controller is far too aggressive and useless in practice. $M_{S, \max }$ is a user selected parameter used for deciding how robust the resulting closed loop system should be. Smaller values gives a less aggressive and more robust controller. The computations needed for correct evaluation of the IAE-related objective function and $M_{S}(\alpha, \lambda)$ have been enabled by correct control laws for systems in innovation form and their closed-loop representation [4].

(26) is a constrained nonlinear optimization problem which is not necessarily convex. Accordingly, we cannot guarantee location of the global optimum of (26 when using solvers such as fmincon, KNITRO, IPOPT, NLOPT, or SNOPT.

\section{Numerical CASE STUdies}

In this section we demonstrate the tuning procedure for simulated case studies. The simulation models considered are the continuous-discrete system

$$
\begin{aligned}
Z(s) & =G(s) U(s)+H(s)(D(s)+W(s)) \\
y\left(t_{k}\right) & =z\left(t_{k}\right)+v\left(t_{k}\right)
\end{aligned}
$$

with the transfer functions

$$
\begin{aligned}
& G(s)=\frac{K}{\tau s+1} e^{-\tau_{d} s} \\
& H(s)=\frac{\bar{K}}{\bar{\tau} s+1} e^{-\bar{\tau}_{d} s}
\end{aligned}
$$

and the parameters $K=20, \tau=10, \bar{K}=-5, \bar{\tau}=2$, $\bar{\tau}_{d}=10$. We consider three different time delays in $G(s)$ : $\tau_{d}=0, \tau_{d}=5$, and $\tau_{d}=20$. In this way, we examine tuning of a system for different ratios between the time constant, $\tau$, and the time delay, $\tau_{d}$. (27a) with (28) is discretized using a zero-order-hold assumption on the inputs $(u, d, w)$ and a sampling time of $T_{s}=1$. With this discretization the system (27) is realized in the state space form (14).The process noise and the measurement noise have the distributions: $w_{k} \sim$ $N_{i i d}(0,0.01)$ and $v_{k} \sim N_{i i d}(0,0.01)$. Independent of $\tau_{d}$, the open-loop output variance of this system is $R_{y y, o l}=0.0712$.

In the synthesis and tuning of the controller, only a model of the transfer function from $\mathrm{U}$ to $\mathrm{Y}, \hat{G}(s)$, is known:

$$
\hat{G}(s)=\frac{\hat{K}}{\hat{\tau} s+1} e^{-\hat{\tau}_{d} s}
$$

The tuning of the controller is conducted using the estimated parameters $\left(\hat{K}, \hat{\tau}, \hat{\tau}_{d}\right)$ in the controller design and an ensemble of parameters to represent the system. The ensemble parameters are perturbation of $\hat{K}, \hat{\tau}$, and $\hat{\tau}_{d}$. The controller tuning is conducted by solving (26) for $M_{S, \max }=$ $\{1.4,1.7,2.0\}$ and using the bounds $\lambda_{\min }=10^{-6}$ and $\lambda_{\max }=10^{6}$. To have offset-free control in this procedure, it is important that the ensemble contains gains and time constants that are different from the gain and time constants of the model used in the design of the controller. In the simulation in this papers, the ensemble consisted of a single gain deviation of $50 \%$.

For evaluation of the resulting closed-loop controller performance with the computed tuning, we have used an MPC as described but with additional input constraints: $-1 \leq u_{k} \leq$ 1. For such closed-loop simulation the true model (27) is also used to represent the system.

\section{A. Case I: No time delay, $\tau_{d}=0$}

In Case I, there is no time delay, i.e. $\tau_{d}=0$. The controllers generated by solution of (26) for this system are listed in Table I. In this case, the difference of the closedloop output variance, $R_{y y, c l}$, for the three controllers is insignificant. The lowest output variance, $R_{y y, c l}$, is obtained with $M_{S, \max }=1.4$. The closed loop output variance is for all controllers smaller than open loop variance. It is also evident from Table I, that the variance of the control signal is dependent of $M_{S, \max }$.

The designed controllers are investigated in terms of their sensitivity functions and by closed-loop simulation. The simulation profile features a reference step from 0 to 5 at $t=0$. A step disturbance enters the system at $t=$ 100. Fig. 2 illustrates the sensitivity function as well as the closed-loop simulation. For all three controllers similar output variance is obtained and the tracking characteristics 
TABLE I: Controller tunings as well as closed loop output and input variance $\left(\tau_{d}=0\right)$

\begin{tabular}{|c|c|c|c|c|}
\hline$M_{S, \max }$ & $\alpha$ & $\lambda$ & $R_{y y, c l}$ & $R_{u u, c l}$ \\
\hline 1.4 & 0.9042 & 2.41 & 0.0634 & 0.0053 \\
\hline 1.7 & 0.9039 & 0.26 & 0.0642 & 0.0119 \\
\hline 2.0 & 0.9038 & 0.0048 & 0.0668 & 0.0168 \\
\hline
\end{tabular}

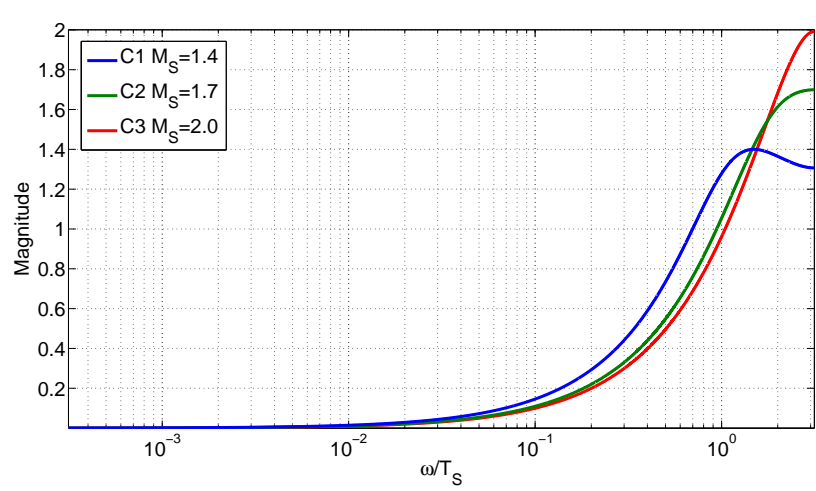

(a) Sensitivity function, $S\left(e^{j \omega T_{s}}\right)$
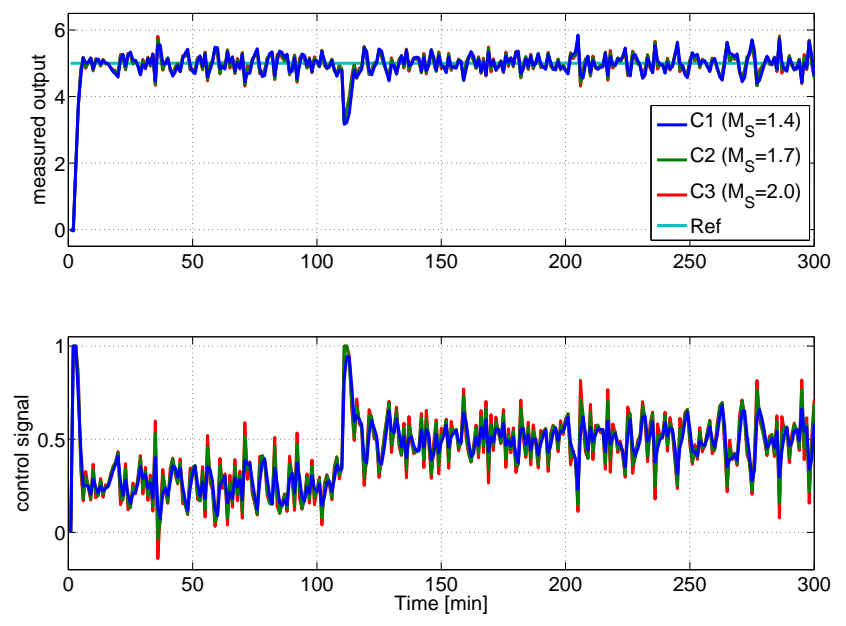

(b) Closed loop simulation

Fig. 2: Sensitivity and simulation results $\left(\tau_{d}=0\right)$

seems indistinguishable. The sensitivity plots show that all three controllers have similar suppression of low frequency noise and disturbances. This is verified in the simulation, where the disturbance is very efficiently rejected for all three controllers. The peak values of all three sensitivity functions occurs close to the Nyquist frequency.

From the simulations, we can conclude that there is no apparent benefit from selecting a high value of $M_{S, \max }$ as this primarily increases output and control signal variance and do not offer a significant better tracking for the system.

\section{B. Case II: Moderate time delay, $\tau_{d}=5$}

In Case II, a moderate time delay, $\tau_{d}=5$, is present in the system. Table II lists the controllers generated for this system by solution of (26). For this system, none of the generated controllers are able to obtain output variance smaller than the
TABLE II: Controller tunings as well as closed loop output and input variance $\left(\tau_{d}=5\right)$

\begin{tabular}{|c|c|c|c|c|}
\hline$M_{S, \max }$ & $\alpha$ & $\lambda$ & $R_{y y, c l}$ & $R_{u u, c l}$ \\
\hline 1.4 & 0.0000 & $2.49 \cdot 10^{4}$ & 0.0811 & $2.04 \cdot 10^{-4}$ \\
\hline 1.7 & 0.9414 & 59.22 & 0.0877 & $6.54 \cdot 10^{-4}$ \\
\hline 2.0 & 0.9048 & $2.2 \cdot 10^{-6}$ & 0.1287 & 0.0169 \\
\hline
\end{tabular}

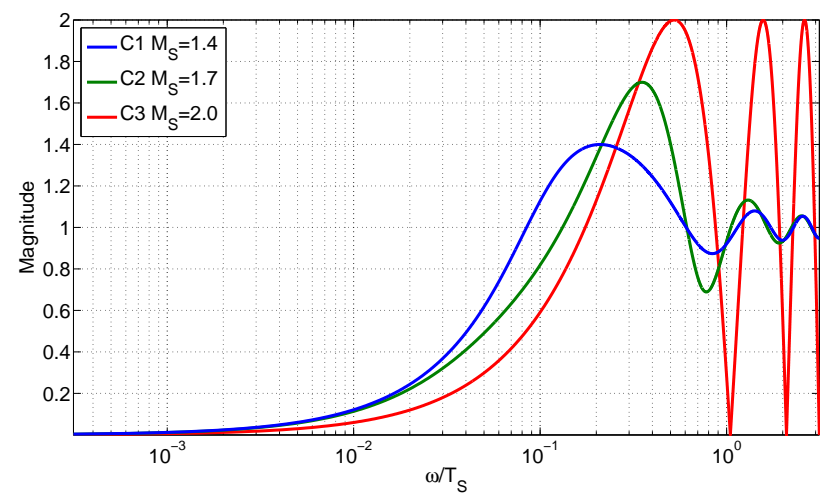

(a) Sensitivity function, $S\left(e^{j \omega T_{s}}\right)$
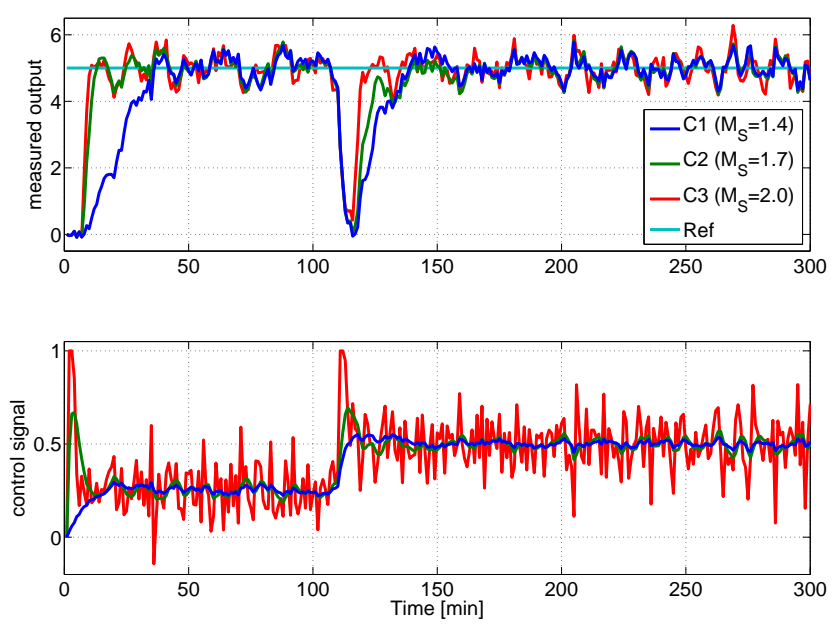

(b) Closed loop simulation

Fig. 3: Sensitivity and simulation results $\left(\tau_{d}=5\right)$

open loop output variance. $R_{y y, c l}$ is dependent on $M_{S, \max }$ and the lowest value is obtained with $M_{S, \max }=1.4$. The tuning with $M_{S, \max }=1.4$ has $\alpha=0$ which corresponds to full integration.

Fig. 3 plots the sensitivity function of the closed loop systems and provides closed-loop simulations for the three controller tunings. The sensitivity plots indicates the controllers for this system deviates from zero at a lower frequency compared to the system without time delay. The sensitivity function for the controller with $M_{S, \max }=2$ is closest to zero in the low frequency region. This characteristic is apparent in the simulations as the controller offers the fastest disturbance rejection. However, this disturbance rejection comes at the price of higher input variations and sensitivity to model-plant mismatch. Therefore, the best trade-off between low input 
TABLE III: Controller tunings as well as closed loop output and input variance $\left(\tau_{d}=20\right)$

\begin{tabular}{|c|c|c|c|c|}
\hline$M_{S, \max }$ & $\alpha$ & $\lambda$ & $R_{y y, c l}$ & $R_{u u, c l}$ \\
\hline 1.4 & 0.9739 & $1.14 \cdot 10^{-6}$ & 0.0812 & 0.0021 \\
\hline 1.7 & 0.9435 & $1.00 \cdot 10^{-6}$ & 0.1027 & 0.0068 \\
\hline 2.0 & 0.9048 & $1.39 \cdot 10^{-6}$ & 0.1414 & 0.0169 \\
\hline
\end{tabular}

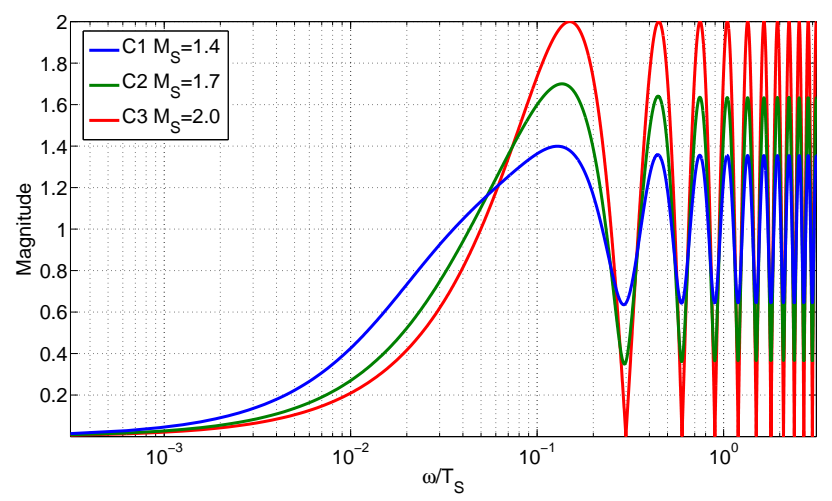

(a) Sensitivity function, $S\left(e^{j \omega T_{s}}\right)$
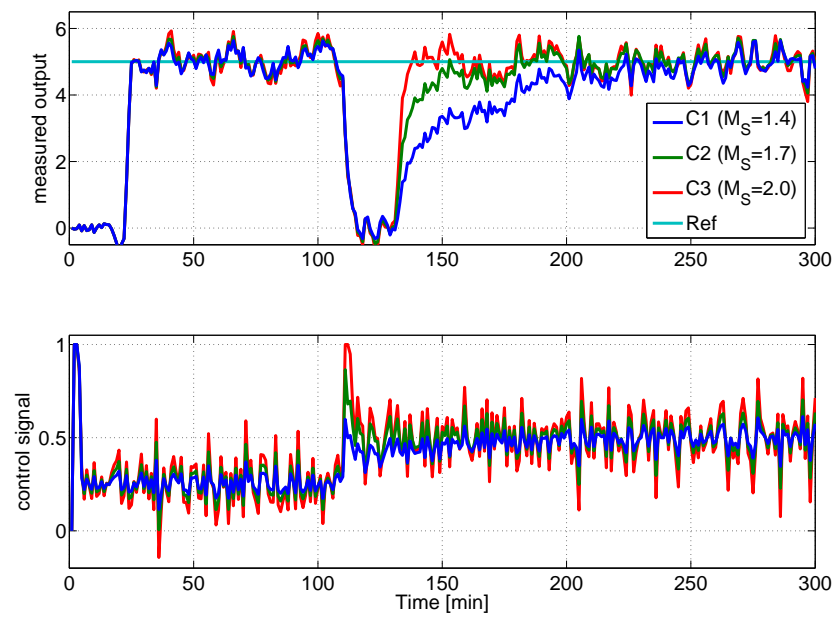

(b) Closed loop simulation

Fig. 4: Sensitivity and simulation results $\left(\tau_{d}=20\right)$

variance and disturbance rejections seems to be obtained for a tuning using $M_{S, \max }=1.4$ or $M_{S, \max }=1.7$.

\section{Case III: Long time delay, $\tau_{d}=20$}

In Case III, the system has a long time delay, $\tau_{d}=20$. The controllers designed by solution of (26) for this system are summarized in Table III. All three controllers have higher output variance than the open loop system. The lowest output as well as input variance is obtained for the tuning using $M_{S, \max }=1.4$.

Fig. 4. plots the sensitivity functions and closed loop simulation for the three controllers. As expected, the long time delay lowers the bandwidth of efficient disturbance rejection. This indicated by the sensitivity function having larger magnitudes at lower frequencies compared to the pre- vious cases. The sensitivity plot for $M_{S}=2.0$ lies below the plots for $M_{S}=\{1.4,1.7\}$ in the low frequency region. This gives a better suppression for low frequency disturbances as reflected in the simulation. However, at higher frequencies this sensitivity function has larger magnitudes which is manifested by the higher input variation revealed in the closed loop simulation. Even for challenging systems with long time delays, the procedure seems to give reasonable tunings based on selection of $M_{S, \max }$.

\section{CONCLUSion}

In this paper, we have presented a procedure to tune an ARX based MPC for univariate processes. The ARX-based MPC has been designed such that it gives offset-free control for type I disturbances (steps). When offset free control is desired, the tuning is non-trivial. The model used by the ARX based MPC is a state space model in innovation form. For such systems, we state the correct control law and develop a state space representation of the closed-loop system. This state space representation can be used for computation of covariances, the sensitivity function, and measures related to the integrated absolute error for deterministic tracking and disturbance rejection scenarios. The suggested tuning is obtained by minimizing a measure related to the integrated absolute error for a set point change scenario. Robustness of the resulting tuning is obtained by restricting the maximum of the sensitivity function by an upper bound. The method has been demonstrated for a first order process with three different dead times.

The only user determined parameter for the tuning is the robustness constraint, $M_{S, \max }$. We have shown how a trade off can be made using this parameter between variance and tracking performance.

\section{REFERENCES}

[1] S. J. Qin and T. A. Badgwell, "A survey of industrial model predictive control technology," Control Engineering Practice, vol. 11, pp. 733764, 2003.

[2] J. K. Huusom, N. K. Poulsen, S. B. Jørgensen, and J. B. Jørgensen, "Tuning of methods for offset free MPC based on ARX model representations," in 2010 American Control Conference, 2010, pp. 2355-2360.

[3] — "Noise modelling and MPC tuning for systems with infrequent step disturbances," in Proceedings of the 18. IFAC World Congress, 2011, pp. $11226-11232$.

[4] J. B. Jørgensen, J. K. Huusom, and J. B. Rawlings, "Finite horizon MPC for systems in innovation form," in 50th IEEE Conference on Decision and Control and European Control Conference, 2011, pp. 1896-1903.

[5] J. K. Huusom, N. K. Poulsen, S. B. Jørgensen, and J. B. Jørgensen, "Tuning SISO offset-free model predictive control based on ARX models," Journal of Process Control, vol. 22, pp. 1997-2007, 2012.

[6] J. L. Garriga and M. Soroush, "Model predictive control tuning methods: A review," Ind. Eng. Chem. Process Design and Developement, vol. 49, pp. 3505-3515, 2010.

[7] J. M. Maciejowski, Predictive Control with Constraints. Prentice Hall, 2002.

[8] R. R. Bitmead and M. Gevers, Adaptive Optimal Control. The Thinking Man's GPC. Prentice Hall International, 1990.

[9] J. H. Lee and Z. H. Yu, "Tuning of model predictive controllers for robust perfomance," Computers in Chemical Engineering, vol. 18, pp. 15-37, 1994.

[10] S. Skogestad and I. Postlethwaithe, Multivariable Feedback Control Analysis and Design. Wiley, 2005. 